\title{
Isolated Congenital Absence of Sternum in a Newborn
}

A 5-day-old boy, born at term (Birth weight $2.52 \mathrm{~kg}$ ), presented to our institution with a congenital defect in the anterior chest wall (Fig. 1). On examination, there was no sternum and the contracting heart with great vessels was visible underneath a thin layer of skin (Web Video 1). A diagnosis of complete sternal cleft was confirmed by digital $X$-rays and computed tomography. No other abnormality was detected.

Asternia (absence of sternum) is rare, and may be associated with Cantrell's pentalogy and Leiber's syndrome. Mother should take adequate precaution while handling such infant during feeding and routine care as there is chance of injuring the heart. The treating physician should also be careful before putting the stethoscope or echocardiography probe over the pulsating heart to avoid external trauma. Surgical repair is the treatment of choice.

*RAKesh Mondal, ARChan Sil AND \# Departments of Pediatric Medicine and "Neonatology, Medical College, Kolkata, West Bengal, India. *ivanrakesh2001@gmail.com

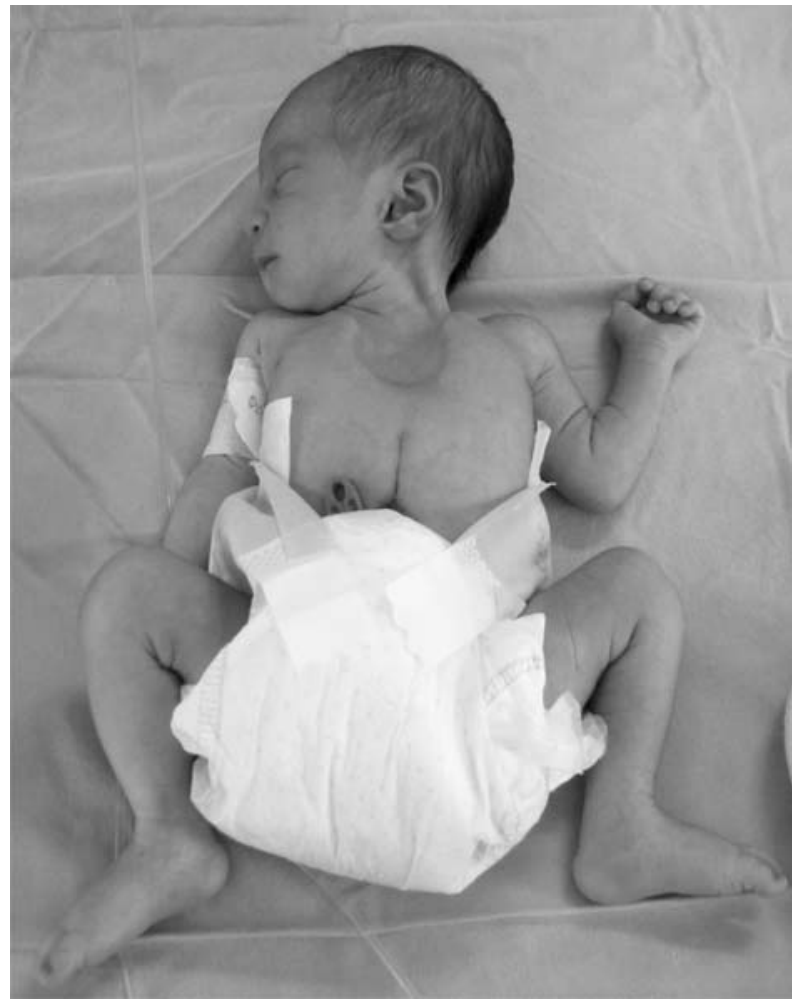

Fig. 1 Congenital absence of sternum in a newborn. (See video of pulsating heart at website).

Notice

\section{Call for Submission of 'Clinical Videos'}

Under this section, Indian Pediatrics publishes videos depicting an intricate technique or an interesting clinical manifestation, which are difficult to describe clearly in text or by pictures. A video file submitted for consideration for publication should be of high resolution and should be edited by the author in final publishable format. MPEG or MP4 formats are acceptable. The maximum size of file should be 20 MB. The file should not have been published elsewhere, and will be a copyright of Indian Pediatrics, if published. For this section, there should be a write-up of up to 250 words discussing the condition and its differential diagnoses. The write up should also be accompanied by a thumbnail image for publication in the print version and PDF. Submit videos as separate Supplementary files with your main manuscript. A maximum of three authors (not more than two from a single department) are permissible for this section. In case the video shows a patient, he/she should not be identifiable. In case the identification is unavoidable, or even otherwise, each video must be accompanied by written permission of parent/guardian, as applicable. Authors are responsible for obtaining participant consent-todisclose forms for any videos of identifiable participants, and should edit out any names mentioned in the recording. The consent form should indicate its purpose (publication in the journal in print and online, with the understanding that it will have public access) and the signed consent of the parent/legal guardian. The copy of the consent form must be sent as supplementary file along with the write-up, and original form should be retained by the author. A sample consent form is available at our website www.indianpediatrics.net. 